\title{
POTENCIAL DIABETOGÊNICO DA STREPTOZOTOCINA EM RATOS: UMA REVISÃO DA LITERATURA
}

\section{DIABETOGENIC POTENTIAL OF STREPTOZOTOCIN IN RATS: A LITERATURE REVIEW}

\author{
Jefferson Matsuiti Okamoto ${ }^{1 *}$, Matheo Augusto Morandi Stumpf ${ }^{1}$, Aryadyne Bueno \\ Rocha Szesz ${ }^{1}$, Vivian Missima Jecohti ${ }^{1}$, Ana Cláudia Garabeli Cavalli Kluthcovsky ${ }^{1}$, \\ Gianna Carla Alberti Schrut ${ }^{1}$. \\ ${ }^{1}$ Universidade Estadual de Ponta Grossa, Departamento de Medicina, Ponta Grossa, \\ Paraná, Brasil. \\ *Autor correspondente: Rua: Casemiro Sinderski, 42- Irati-Pr. E-mail:okamotojeff@gmail. \\ com
}

\section{RESUMO}

Devido ao aumento da prevalência do Diabetes Mellitus em diversos países, faz-se necessária a realização de estudos experimentais para o melhor entendimento da sua fisiopatologia. O método químico com utilização prevalente de estreptozotocina para indução de diabetes mellitus em ratos é um dos mais rápidos e de menor custo, sendo esta técnica o foco do presente trabalho. Através de uma minuciosa revisão de literatura, busca-se as descrições atuais do potencial diabetogênico da streptozotocina em ratos. A busca de referências foi realizada nos portais de periódicos MEDLINE e Scielo entre os meses de junho e agosto de 2016. Utilizou-se as seguintes palavras como descritores, em inglês/português: "streptozotocin; diabetes mellitus; rats". A streptozotocina tem sido utilizada em estudos experimentais para induzir o diabetes em modelos animais, porém percebe-se a necessidade de uma padronização em seu uso para que se obtenham resultados mais efetivos durante as pesquisas. Como o resultado final da ação da streptozotocina depende do modelo animal utilizado e também do modo de preparo, via de administração e dose, devemos recordar que todos esses fatores devem ser levados em consideração quando a pesquisa for iniciada, evidenciando a crucial importância de um melhor entendimento de todos os aspectos do mecanismo de ação da streptozotocina na indução do diabetes. O desenvolvimento de mais estudos com o uso de streptozotocina deve ser encorajado, utilizando-se maior amostragem e estirpes mais sensíveis, para que se possa chegar mais próximo de desvendar a completa fisiopatologia do diabetes.

Palavras-chave: Streptozotocina. Diabetes Mellitus. Ratos.

\begin{abstract}
Due to the increasing prevalence of Diabetes Mellitus disease in several countries, it is necessary to conduct experimental studies to understand all its pathophysiology. One of the cheapest and fastest ways to induce diabetes in murine models is the chemical method using the streptozotocin as the main substance, technique that is the focus of this study. The research was done by a thorough literature review about the current descriptions to the streptozotocin uses and its relationship with the diabetogenic potential. The data basis used were Scielo and MEDLINE jounals web-portals, between June and August 2016, and the keywords (English and Portuguese) were: "streptozotocin; diabetes mellitus; rats". The streptozotocin has widely been using in experimental studies to induce diabetes in animal models. However, it is necessary to have a standardization in its use to achieve the most effective outcome during the research. The results of streptozotocin application depend on the animal model used and also the method of preparation, route of administration and dose. We must consider all of these factors during the experiments, revealing the importance of the knowledge about streptozotocin
\end{abstract}


to induce diabetes. More studies should be done with streptozotocin, using larger sample and more sensitive strains, in order to unfold the full pathophysiology of diabetes.

Keywords: Streptozotocin. Diabetes Mellitus. Rats.

\section{INTRODUÇÃO}

A insulina é um hormônio-chave na absorção de glicose pelas células do corpo. Sua falta absoluta, ou sua ação pouco efetiva na captação de glicose pelas células, pode levar a um quadro hiperglicêmico que, em determinados níveis, caracteriza o diabetes mellitus $(\mathrm{DM})^{1}$. Dados da Federação Internacional do Diabetes demonstram que em 2015 existiam aproximadamente 415 milhões de diabéticos no mundo. A perspectiva é que esse número aumente, sendo que cerca de 642 milhões de pessoas terão a doença até $2040^{2}$.

O diabetes é uma doença que causa preocupação em todo o mundo, mas ainda diversos aspectos relacionados à patogênese do DM permanecem desconhecidos $^{3}$. Tendo em vista a dificuldade do estudo da doença em seres humanos, vários modelos animais têm sido utilizados e se tornaram fundamentais para tentar entender alterações morfológicas, fisiológicas e metabólicas provocadas em diversos tecidos e órgãos ${ }^{4}$. Os animais mais utilizados são os ratos e especificamente se dividem em dois grandes modelos: os ratos experimentalmente induzidos ao diabetes e os genéti$\cos ^{5}$. Os primeiros podem ser induzidos cirurgicamente (pancreatectomia) ou quimicamente ${ }^{5,6}$.

Neste trabalho, haverá enfoque especial aos métodos químicos de indução ao DM, por serem mais simples, baratos e acessíveis ${ }^{5,6}$. A streptozotocina (STZ) é uma das principais substâncias químicas utilizadas devido ao seu grande potencial diabetogênico e pela possibilidade de manutenção do quadro hiperglicêmico em determinadas doses ${ }^{7}$. O objetivo do presente estudo é realizar uma revisão da literatura sobre o potencial diabetogênico da STZ em ratos, com enfoque em seu mecanismo de ação e de sua aplicação na área de pesquisa científica.

\section{MÉTODO}

Trata-se de um estudo de revisão da literatura com instruções acerca da STZ para se analisar sua associação com o potencial diabetogênico. As referências bibliográficas usadas foram os portais de periódicos Medical Literature Analysis and Retrieval System
Online (MEDLINE) e da Scientific Electronic Library Online (Scielo).

Foram acessados os referidos portais, em agosto de 2016, utilizando-se as palavras-chave em inglês e português na seguinte combinação: "streptozotocin; diabetes mellitus; rats", sem limitação de tempo. Ao total foram identificadas 4662 publicações, sendo todas provenientes do portal MEDLINE. Foram incluídos estudos que abordassem a atividade diabetogênica da STZ, selecionando 93 artigos. Desses foram considerados apenas os artigos experimentais que realizassem a indução química in vivo e que instruíssem como foi feita a indução, levando em consideração modo de preparo, via de administração e a relação entre a dose da droga e o tempo de indução, além de fornecer dados como do modelo animal utilizado (espécie sexo, idade, peso e estado nutricional). $\mathrm{O}$ resultado foi de 20 artigos.

\section{RESULTADOS}

A STZ é um derivado da Streptomyces achromogenes, apresentando atividade antibiótica e antineoplásica de amplo espectro, além de sua função diabetogênica $^{8,9}$. Sua estrutura básica é muito semelhante à da glicose, pois ela é facilmente captada pelos transportadores de glicose GLUT- $2^{10}$. Como as células $\beta$ são mais ativas na captação da glicose, os efeitos citotóxicos da STZ também são mais pronunciados nesse local ${ }^{11}$. A ação tóxica da STZ ocorre por ser um agente alquilante do DNA, fazendo com que a enzima poli (ADP-ribose) polimerase se ative e ocorra depleção de NAD+ celular, levando a redução dos níveis de ATP e, consequentemente, inibição da produção e secreção de insulina ${ }^{12}$.

Também foi documentado que a STZ aumenta os níveis de óxido nítrico e de espécies reativas de oxigênio, o que aumenta o dano ao DNA ${ }^{13,14}$. O resultado final da ação da STZ depende do modelo animal utilizado (espécie, sexo, idade, peso e estado nutricional) e também do modo de preparo, via de administração e dose $e^{6,7}$. 
Em geral, a STZ é administrada em baixa temperatura, devido à instabilidade do composto na temperatura ambiente, e em baixo $\mathrm{pH}$, sendo que a máxima estabilidade da droga é conseguida com pH igual a $4^{9,15}$. No entanto, foi relatado que as soluções de STZ com pH mais elevado $(7,2-7,4)$ são tão estáveis como aquelas com um $\mathrm{pH}$ de 4,5 , isso por pelo menos uma hora a $37^{\circ} \mathrm{C}$ e pH 6,7-7,8 durante 30 minutos a $0^{\circ} \mathrm{C}^{6,16}$. A STZ pode ser dissolvida em solução salina acidificada a $0,9 \%$, mas é obtida maior estabilidade da droga em tampão citrato ( $\mathrm{pH} 4,5)$, sendo mais adequada para aplicação ${ }^{16}$.

Existem diversas vias de administração de STZ: intramuscular, subcutânea, intraperitoneal e intravenosa, sendo as duas últimas as mais utilizadas ${ }^{6,17,18,19}$. Recentemente, em um estudo realizado em ratos Wistar-Furth isogênicos, com dose de $60 \mathrm{mg} / \mathrm{kg}$ de STZ, foram avaliadas as vias intraperitoneal e a intravenosa (pela veia caudal e sublingual). Verificou-se que todas as vias geraram níveis semelhantes de severidade e duração do diabetes ${ }^{17}$. Porém, houve $30 \%$ de falha tanto na via intraperitoneal quanto na sublingual, além de $10 \%$ na caudal. Outros estudos, no entanto, obtiveram diabetes duradouro em $100 \%$ dos animais que receberam STZ intraperitonealmente na mesma dose ${ }^{17}$.

Para os estudos utilizando ratos machos, a injeção na veia peniana é uma outra opção, pois a técnica é mais simples e de maior acessibilidade ${ }^{18}$. Dentro de um ciclo circadiano (24hrs), foi verificada a maior incidência de indução do diabetes quando a STZ foi administrada às $16 \mathrm{~h} 00$ e a menor incidência às $08 \mathrm{~h} 00$, indicando um ritmo circadiano ${ }^{19}$.

Em ratos Wistar as doses podem ser dividas em altas (maiores que $65 \mathrm{mg} / \mathrm{kg}$ de peso corporal), intermediárias $(40-55 \mathrm{mg} / \mathrm{kg}$ ) e baixas (menores que 35 $\mathrm{mg} / \mathrm{kg})^{7}$. Uma única dose baixa em ratos não produz efeito significativo e uma dose de $35 \mathrm{mg} / \mathrm{kg}$ cursa com recuperação espontânea do estado diabético em $25 \%$ da amostra .

A doença estável tem sido observada em doses de $55-65 \mathrm{mg} / \mathrm{kg}^{7}$; na dose de $65 \mathrm{mg} / \mathrm{kg}$ é observado um aumento nos níveis glicêmicos duas horas após a injeção de STZ, mas sem qualquer aumento de insulina sérica. Sete horas após a aplicação ocorre hipoglicemia, com aumento significativo de insulina sérica sem diminuição da insulina plasmática; após 24 horas, estabelece-se o quadro hiperglicêmico. Esse estado metabólico se mantém inalterado após 7 e 28 dias, sendo que dados similares são observados nas doses de $55 \mathrm{mg} / \mathrm{kg}^{7}$. Uma dose de $65 \mathrm{mg} / \mathrm{kg}$ independente da via administrada e, em média, induz o diabetes em 14 a 15 dias $^{20,21}$. A lipemia está presente, porém a cetonúria só é obtida com doses maiores que $100 \mathrm{mg} / \mathrm{kg}^{7,20,21}$.

O desenvolvimento da hiperglicemia nesses ratos, após a injeção de STZ, é consequente da destruição direta das células $\beta$ pancreáticas e resulta na deficiência de insulina ${ }^{22}$. Desta forma, a hiperglicemia observada não é decorrente de um quadro de resistência insulínica. Assim sendo, para se chegar mais próximo de um modelo diabético tipo 2, faz-se necessário um quadro de resistência insulínica e deficiência na produção de insulina pelas células $\beta$, o que pode ser conseguido com a utilização baixas doses de STZ e dieta hiperlipídica ${ }^{22}$.

A sensibilidade à STZ é altamente variável em ratos, dependendo da estirpe do animal. Dados mostram que o grau de sensibilidade para STZ, medido pelo nível de glicose no sangue, é DBA/2J $>$ C57BL/6J $>\mathrm{MRL} / \mathrm{MpJ}>129 / \mathrm{SvEv}>\mathrm{BALB} / \mathrm{c}^{23}$ - essas siglas correspondem à nomenclatura utilizada para definir as linhagens consanguíneas dos ratos. Pode-se ainda separar essas estirpes em grupos de baixos respondedores (MRL/ MpJ, BALB/c, e 129/SvEv) e altos respondedores (C57BL/6J e DBA/2J) à STZ.

Em outro estudo foi observado que a menor dose única de STZ necessária para induzir diabetes em ratos C57BL/6J, ICR, ddY e BALB/c é de 100, 100, 125 e $150 \mathrm{mg} / \mathrm{kg}$, respectivamente ${ }^{24}$. Embora existam essas tendências na sensibilidade dependente da estirpe, alguns subgrupos podem experimentar sensibilidades diferentes para STZ.

Comparadas a ratos machos, as fêmeas são menos afetadas pelos efeitos da STZ, principalmente devido à atividade anti-apoptótica do estradiol ${ }^{25}$. Em experiências com ratos C57BL/KsJ foi relatada uma diferença altamente significativa entre os valores médios de glicose sanguínea, sendo que a média de glicemia era $200 \mathrm{mg} / \mathrm{dL}$, superior nos machos em relação às fêmeas, 35 dias após a injeção de $\mathrm{STZ}^{25}$.

Quanto ao estado alimentar, protocolos como os do National Institutes of Health (NIH) recomendam que o rato esteja em jejum de pelo menos quatro horas antes do tratamento por $\mathrm{STZ}^{26}$. Esse método é justificado, pois acredita-se que a glicose elevada possa competir pelos receptores GLUT-2 com a STZ, diminuindo sua ação diabetogênica ${ }^{10}$. Contudo, foi verificado recentemente que a alimentação não causa alterações 
na efetividade da indução da STZ à intolerância a glicose, hiperglicemia, disfunção ou perda de células $\beta$. Com isso, as repetidas rodadas de jejum/realimentação necessárias para protocolos de administração em ratos de laboratório acabam sendo somente estressantes para o animal e, portanto, desnecesárias ${ }^{26}$.

As doses de indução da STZ e seu mecanismo de ação já são bem descritos na literatura. Pode-se dizer, então, que se trata de uma droga segura e efetiva de se induzir diabetes em ratos, devendo ser utilizada para tal finalidade. Estudos recentes que utilizam essa maneira de indução objetivam muitas vezes entender a fisiopatologia do DM, ainda obscura.

\section{CONCLUSÃO}

A utilização do STZ é uma maneira relativamente rápida de se induzir o DM, tanto tipo 1 quanto 2, em experimentos com ratos. É importante que o pesquisador saiba sua dosagem e que ela varia entre sexos de animais e estirpes distintas. Com essas informações, há menor risco de imperícia numa futura pesquisa.

Sugere-se que sejam realizados estudos com estirpes mais sensíveis à STZ e que avaliem características do DM. que ainda não se tem conhecimento, como o grau de hiperplasia e hipertrofia intestinal, mudança de microbiota, entre outras alterações histológicas diversas que se deseje investigar.

\section{REFERÊNCIAS}

1. Ozougwu JC, Obimba, KC, Belonwu CD, Unakalamba $\mathrm{CB}$. The pathogenesis and pathophysiology of type 1 and type 2 diabetes mellitus. J. Physiol. Pathophysiol. 2013; 4(4): 46-57.

2. International Diabetes Federation. IDF Diabetes Atlas $7^{\text {th }}$ Ed. [Internet]. [Acesso em 10 de agosto de 2016]. Disponível em: <http://www.diabetesatlas.org $>$.

3. Baynest, HW. Classification, Pathophysiology, Diagnosis and Management of Diabetes Mellitus. J Diabetes Metab 2015; 6(5): 1-9.

4. King AJ. The use of animal models in diabetes research. $\mathrm{Br}$ J Pharmacol. 2012; 166: 877-894.

5. Islam MS, Wilson RD. Experimentally induced rodent models of type 2 diabetes. Methods Mol Biol. 2012; 933: 161-74.

6. Deeds MC, Anderson JM, Armstrong AS, Gastineau DA, Hiddinga HJ, Jahangir A, et al. Single dose streptozotocininduced diabetes: considerations for study design in islet transplantation models. Lab. Anim 2011; 45: 131-140.
7. Junod A, Lambert AE, Stauffacher W, Renold AE. Diabetogenic action of streptozotocin: relationship of dose to metabolic response. Journal of Clinical Investigation 1969; 48(11): 2129-2139.

8. Herr RR, Eble TE, Bergy ME, Jahnke HK. Isolation and characterization of streptozotocin. Antibiot. Annu 1960; 7 : 236-240.

9. Bono VHJ. Review of mechanism of action studies of the nitrosoureas. Cancer Treat Rep 1976; 60(6): 699-702.

10. Schnedl WJ, Ferber S, Johnson JH, Newgard CB. STZ transport and cytotoxicity: specific enhancement in GLUT2expressing cells. Diabetes 1994; 43: 1326-1333.

11. Tjälve H, Wilander E, Johansson E. Distribution of labelled streptozotocin in mice: uptake and retention in pancreatic islets. J. Edocr 1976, 69: 455-456.

12. Elsner M, Guldbakke B, Tiedge M, Munday R, Lenzen S. Relative importance of transport and alkylation for pancreatic beta-cell toxicity of streptozotocin. Diabetologia 2000; 43: 1528-1533.

13. Kröncke KD, Fehsel K, Sommer A, Rodriguez ML, Kolb-Bachofen V. Nitric oxide generation during cellular metabolization of the diabetogenic N-methyl-N-nitrosourea streptozotocin contributes to islet cell DNA damage. Chem Hoppe-Seyler 1995; 376: 179-185.

14. Bedoya FJ, Solano F, Lucas M. N-monomethylarginine and nicotinamide prevent streptozotocin-induced double strand DNA break formation in pancreatic rat islets. Experienti 1996; 52: 344-347.

15. Srinivasan K, Viswanad B, Asrat L, Kaul CL, Ramarao P. Combination of high-fat diet-fed and low-dose streptozotocin-treated rat: A model for type 2 diabetes and pharmacological screening. Pharmacol Res. 2005; 52(4): 313-320.

16. Lenzen S. The mechanisms of alloxan- and streptozotocin-induced diabetes. Diabetologia 2008; 51: 216-226.

17. Delfino VDA, Figueiredo JF, Matsuo T, Favero ME, Matni AM, Mocelin AJ. Streptozotocin-induced diabetes mellitus: long-term comparison of two drug administration routes. J. bras. nefrol 2002; 24(1): 31-36.

18. Evan AP, Mong SA, Gattone VH, Connors BA, Aronoff GR, Luft FC. The effect of streptozotocin and streptozotocininduced diabetes on the kidney. Renal physiology 1984; 7(2): 78-89.

19. Candela S, Hernandez RE, Gagliardino JJ. Circadian variation of the streptozotocin-diabetogenic effect in mice. Experientia 1979; 35: 1256-1257. 
20. Rabelo SB, Villaverde AGJB, Nicolau RA, Salgado MAC, Melo MS, Pacheco MTT. Comparison between the wound healing at induced diabetic and non-diabetic rats after LLLT. J. Clin Laser Med. Surg 2006; 24: 474-479.

21. Reddy GK. Comparison of the photostimulatory effects of visible $\mathrm{He}-\mathrm{Ne}$ and infrared Ga-As lasers on healing impaired diabetic rat wounds. Lasers Surg. Med 2003; 33(5): 344-351.

22. Qinna NA, Badwan AA. Impact of streptozotocin on altering normal glucose homeostasis during insulin testing in diabetic rats compared to normoglycemic rats. Drug Des Devel Ther. 2015; 9: 2515-2525.

23. Gurley SB, Clare SE, Snow KP, Hu A, Meyer TW, Coffman TM. Impact of genetic background on nephropathy in diabetic mice. American journal of physiology 2006; 290(1): 214-222.

24. Hayashi K, Kojima R, Ito M. Strain differences in the diabetogenic activity of streptozotocin in mice. Biological \& pharmaceutical bulletin 2006; 29(6): 1110-1119

25. Leiter EH. Multiple low-dose streptozotocin-induced hyperglycemia and insulitis in C57BL mice: influence of inbred background, sex, and thymus. Proceedings of the National Academy of Sciences of the United States of America 1982; 79(2): 630-634.

26.Chaudhry ZZ, Morris DL, Moss DR, et al. Streptozotocin is equally diabetogenic whether administered to fed or fasted mice. Laboratory animals 2013; 47(4): 257-265. 